\title{
Editorial
}

\section{Hemodynamic Aspect of Extracorporeal Circulation}

\author{
Hisao Manabe, M. D.
}

$\mathbf{R}^{\text {ECENT advances in the developement of pump oxygenator and its }}$ R clinical application for the maintenance of circulation and respiration enabled a surgeon to perform intracardiac operation with safe. First successful intracardiac operation with the aid of extracorporeal circulation was performed in 1954. The length of perfusion, however, in which heart could be opened was short and insufficient for the correction of the complicated intracardiac lesions. Since then, numerous efforts were paid for the purpose to prolong the length of perfusion without producing any deleterious effects upon the patient undergoing open heart surgery. Most of these efforts, however, were carried out in the metabolic aspect of extracorporeal circulation and, on the contrary, little efforts were paid in the hemodynamic aspect of this technique. Hemodynamic problems such as disturbances in blood balance, blood distribution and peripheral blood flow were recognized only when metabolic abnormalities became evident as a result of these disturbances after prolonged extracorporeal circulation.

For the last decade, we have devoted ourselves to the study of hemodynamic changes associated with extracorporeal circulation for the purpose of establishing a method for prolonged extracorporeal circulation so that we can perform the complicated intracardiac operations without haste. Developement of self-balancing method, determination of optimum flow rate and maintenance of effective peripheral blood flow during cardiotomy are those problems with the solutions of which we have established our present method of extracorporeal circulation, with the application of which patient could survive after 6 hours of cardiopulmonary bypass. Following are those three hemodynamic problems which we believe very important for the adequate maintenance of extracorporeal circulation.

\section{Developement of Self-Balancing Method}

Maintenance of adequate capillary blood flow is fundamental in extracorporeal circulation. Blood flow through arterioles and venous pressure

Professor of Surgery, Osaka University Medical School, Osaka. 
are the factors which affect the capillary blood flow. As the former depends upon the pump output during perfusion, capillary blood flow is related mainly to the venous pressure. In other words, the maintenance of venous pressure is important for the maintenance of blood flow through capillary during extracorporeal circulation.

Relationship between central venous pressure, size of drainage catheters inserted into venae cavae, and blood flow through the catheters were calculated through hydraulics and also studied experimentally. Based on these results, an adequate method for the maintenance of venous pressure during extracorporeal circulation was established. With this method, the same amount of blood with the venous return of patient automatically drain into the pump oxygenator throughout the duration of cardiopulmonary bypass. This method is fundamental for the maintenance of extracorporeal circulation depending upon the autoregulatory mechanism of the patient undergoing perfusion and was named as "self-balancing method".

\section{Determination of Optimum Flow Rate}

A relatively short period of extracorporeal circulation can be maintained safely with wide range of perfusion flow rate when above mentioned " selfbalancing method" was utilized. However, to keep the patient's circulatory condition unchanged for the prolonged period of extracorporeal circulation by his own control mechanism, it is necessary to adopt a flow rate which keeps the state of the peripheral vascular bed unchanged.

Hemodynamics during extracorporeal circulation was studied for the purpose of determination of such "an optimum flow rate" during perfusion. No close relationship was found between blood flow and blood pressure during extracorporeal circulation. However, so long as blood flow was kept higher than 60 per cent of the preoperative cardiac output, blood pressure was always maintained above the so-called shock level. The relationship between blood flow rate and total peripheral resistance during extracorporeal circulation was similar to that obtained preoperatively between cardiac output and total peripheral resistance. The ratio of peripheral vascular resistance during cardiopulmonary bypass to that before operation (resistance ratio) was plotted against the ratio of blood flow during cardiopulmonary bypass to cardiac output before operation (perfusion ratio). An inverse relationship was observed between these two ratios. With a perfusion ratio higher than 0.6 , the resistance ratio was usually lower than 1.0. With continuance of cardiopulmonary bypass with this flow rate, the blood shifted from the pump oxygenator to the patient to compensate for vasodilatation. On the contrary, with a perfusion ratio lower than 0.6 , the resistance ratio was kept usually higher than 
1.0. When the perfusion is continued with this flow rate, the patient's blood is squeezed out and the flow into parts of organs or tissues will be reduced because of vasoconstriction. In such a case the blood will be shifted from the patient to the pump oxygenator. When the flow ratio was close to 0.6 , total peripheral resistance was kept at a preoperative level. The blood volume within the patient with this perfusion ratio was automatically kept unchanged at the termination of cardiopulmonary bypass. This flow rate was about $2.2 \mathrm{~L}$. per min. per M. ${ }^{2}$ and was considered as "optimum flow rate" in maintaining cardiopulmonary bypass.

\section{Maintenance of Effective Peripheral Blood Flow}

During clinical perfusion with cardiotomy, caution must be paid to the fact that the effective peripheral blood flow sometimes much decreased even though the above mentioned optimum flow rate was utilized. Intracardiac return during cardiotomy differs from case by case and depends upon type and severity of cardiac lesions. This is composed of coronary blood flow and bronchial blood flow and is considered to be uneffective flow for the peripheral vascular bed. Particularly large amount of bronchial blood flow was noted in case with tetralogy of Fallot. The rapid and severe increase of coronary blood flow was cncountered after the end of anoxic cardiac arrest. In such a case the peripheral blood flow decreased if the pump output was kept unchanged. The use of an electromagnetic flowmeter to measure the caval return instantaneously proved to be helpful in maintaining the blood flow through peripheral vascular bed at constant optimum level regardless of the amount of intracardiac return even in such cases mentioned above.

Those three are the major problems, the solution of which was fundamental for the safe maintenance of prolonged extracorporeal circulation. Thus the open heart surgery is now carried out with almost no limit in time as a result of these investigations.

\section{REFERENCES}

1. Kawashima, Y., Fujita, T., Fujimoto, K., and Manabe, H. : J. Thorac. Cardiovas. Surg. 48: 1007, 1964.

2. Manabe, H., Kawashima, Y., Fujita, T., Ueda, T., Yamamoto, Z., and Fujimoto, K.: J. Thorac. Cardiovas. Surg. 51 : 213, 1966. 